\title{
Fabrication of isotropic bulk graphite using artificial graphite scrap
}

\author{
Sang-Min Lee ${ }^{1}$, Dong-Su Kang ${ }^{1}$, Woo-Seok Kim ${ }^{2}$ and Jea-Seung Roh ${ }^{1, *}$ \\ ${ }^{1}$ School of Advanced Materials and Systems Engineering, Kumoh National Institute of Technology, Gumi 730-701, Korea \\ ${ }^{2}$ Carbolab Co., Ltd., Gumi 730-701, Korea
}

\section{Article Info}

Received 22 January 2014

Accepted 2 April 2014

*Corresponding Author

E-mail: jsroh@kumoh.ac.kr

\section{Open Access}

DOI: http://dx.doi.org/

10.5714/CL.2014.15.2.142

This is an Open Access article distributed under the terms of the Creative Commons Attribution Non-Commercial License (http://creativecommons.org/licenses/ by-nc/3.0/) which permits unrestricted non-commercial use, distribution, and reproduction in any medium, provided the original work is properly cited.

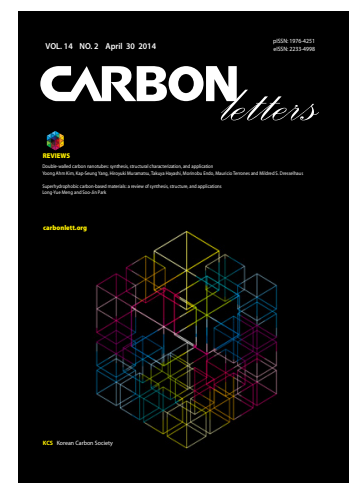

http://carbonlett.org

pISSN: 1976-4251

elSSN: 2233-4998

Copyright $\odot$ Korean Carbon Society

\begin{abstract}
Isotropic synthetic graphite scrap and phenolic resin were mixed, and the mixed powder was formed at $300 \mathrm{MPa}$ to produce a green body. New bulk graphite was produced by carbonizing the green body at $700^{\circ} \mathrm{C}$, and the bulk graphite thus produced was impregnated with resin and re-carbonized at $700^{\circ} \mathrm{C}$. The bulk density of the bulk graphite was $1.29 \mathrm{~g} / \mathrm{cm}^{3}$, and the porosity of the open pores was $29.8 \%$. After one impregnation, the density increased to $1.44 \mathrm{~g} / \mathrm{cm}^{3}$ while the porosity decreased to $25.2 \%$. Differences in the pore distribution before and after impregnation were easily confirmed by observing the microstructure. In addition, by using an X-ray diffractometer, the degrees-of-alignment (Da) were obtained for one side perpendicular to the direction of compression molding of the bulk graphite (the "top-face"), and one side parallel to the direction of compression molding (the "side-face"). The anisotropy ratio calculated from the Da-values obtained was 1.13 , which indicates comparatively good isotropy.
\end{abstract}

Key words: isotropic synthetic graphite scrap, bulk graphite, impregnation, degree of alignment, anisotropy ratio

\section{Introduction}

Because graphite has an anisotropic crystal structure and boasts far better heat resistance, corrosion resistance, electric conductivity, high-temperature strength, and lubricity than other materials, it is used in diverse fields. These include use in high-temperature structural materials such as electrodes, carbon brushes, mechanical seals and special mechanical parts [1-3]. When graphite with such an anisotropic structure is formed using the cold isostatic pressing method, graphite particles are arranged in a disorderly manner, exhibit isotropy, have a dense structure, and are high in density and strength. In addition, because the green body has no directionality, its physical and electrical characteristics are uniform in all directions (isotropic) [4,5].

In the general production process for an isotropic, synthetic, graphite block; petroleumor tar-pitch-derived coke powder is evenly mixed with a binder such as pitch or resin, after which it is formed and then carbonized. At this stage, because of the volatilization of the binder due to carbonization, pores are generated inside the green body, and the green body is impregnated with pitch or resin to fill the pores. After filling the pores by several impregnation cycles, the green body is graphitized by thermal treatment at $2500^{\circ} \mathrm{C}$ or above [6-8].

After being produced through such a complicated process, the isotropic, synthetic, graphite block is processed for a specific usage, and a large amount of scrap (ISGS) is generated. The graphite scrap generated during the process is added to molten iron to increase the carbon content at steel mills or used to produce graphite-added refractory materials. In particular, when producing carbon-magnesite bricks, approximately $15-25 \%$ graphite scrap is added. However, the overall amount of graphite scrap used is not large, and most of it is 
discarded [9-11].

Consequently, the present study sought to produce isotropic bulk graphite using ISGS. Phenolic resin was used as the binder, and changes in the density and porosity were measured after a single impregnation of the recycled isotropic bulk graphite. In addition, using X-ray diffractometer (XRD), the degrees-ofalignment $(\mathrm{Da})$ and microstructure according to the direction of compression molding were measured, and the anisotropy ratio was obtained from the Da. The results of the present study were used to evaluate the potential of producing isotropic bulk-graphite from ISGS, and to provide data for future production of highdensity, isotropic bulk-graphite.

\section{Experimental Procedure}

\subsection{Raw materials and preparation}

The raw powder used in the present study was ISGS remaining after processing of isotropic bulk graphite for machining [12]. The particle size of the raw powder was measured using a particle size analyzer (Malvern Ins. GB/Mastersizer 2000). The binder was phenolic resin produced by Kangnam Chemical Co., Ltd. The mixture of the raw material and the binder was subjected to uniaxial press-forming at a pressure of $300 \mathrm{MPa}$, thus producing a green body with a diameter of $10 \mathrm{~mm}$. The green body thus formed was carbonized for one hour at $700^{\circ} \mathrm{C}$ in nitrogen gas. The pores generated by the carbonization process were re-carbonized after being resin-impregnated one time.

\subsection{Density and porosity measurement}

Changes in the density and porosity of the recycled bulk graphite, before and after impregnation, were measured using the Archimedes method (ISO 18754: 2003). The density measured in the present study is volumetric density, which includes both open and closed pores; and the porosity was obtained from measurement of the volume of open pores. Here, 'open pores' refers to penetrating pores and ink-bottle pores through which fluids can infiltrate $[13,14]$.

\subsection{Observations of microstructure}

The bulk graphite produced was ground using sandpaper (\#1200-\#2400) and ultimately micro-ground at $0.25 \mu \mathrm{m}$. An optical microscope (Nikon Eclipse, LV150) was used to examine the microstructure of the bulk graphite before and after impregnation. One side perpendicular to the direction of compression molding (henceforth "top-face"), and one side parallel to the direction of compression molding (henceforth "side-face"), was examined.

\subsection{XRD analysis}

To confirm the Da of the recycled bulk graphite, an XRD (SWXD, X-MAX/2000-PC, Rigaku) analysis was conducted. The wavelength $\left(\mathrm{Cu}-\mathrm{K}_{1}\right)$ of the X-ray target used was 1.5406 $\AA$, and XRD spectra were obtained using the $2 \theta$ continuous scanning method at a scanning rate of $1 \% \mathrm{~min}$, within a scan- ning range of $10-60^{\circ}$. Using the XRD data, the top-face and the side-face were measured, and the Da values were compared. The Da values were calculated according to the following equation, using the values of relative strength divided by the height at the (100)-peak and at the (002)-peak. Below, $\mathrm{I}_{002}$ and $\mathrm{I}_{100}$ are the height at the (002)-peak and the (100)-peak, respectively $[15,16]$ :

$$
\mathrm{Da}=\mathrm{I}_{100} /\left(\mathrm{I}_{100}+\mathrm{I}_{002}\right)
$$

In addition, the anisotropy ratio was obtained from the Da ratio $\left(\mathrm{Da}^{\mathrm{Top}} / \mathrm{Da}^{\mathrm{Side}}\right)$ of the top-face and the side-face [17-19].

\section{Results and Discussion}

\subsection{ISGS powder analysis}

Fig. 1 shows the results of the particle-size analysis of ISGS and the XRD spectra. The average particle size in the raw powder was approximately $50 \mu \mathrm{m}$. In the XRD spectra, the strength

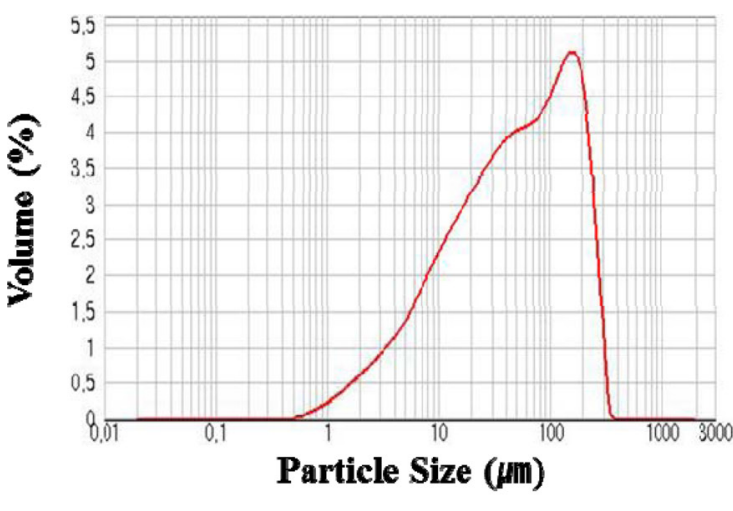

$d(0.1)=6.254 \mu m ; d(0.5)=50.034 \mu m ; d(0.9)=193.206 \mu m$

(a)

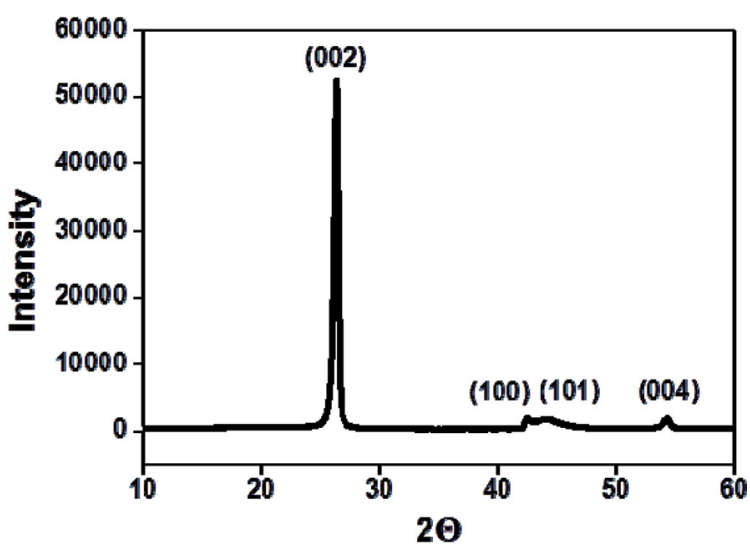

(b)

Fig. 1. Isotropic synthetic graphite scrap results: (a) particle-size analysis and (b) X-ray diffractometer spectrum. 
Table 1. Result of bulk density and porosity (open pore) measurement

\begin{tabular}{|c|c|c|c|c|c|}
\hline Status & Sample & $\begin{array}{l}\text { Bulk density } \\
\qquad\left(\mathrm{g} / \mathrm{cm}^{3}\right)\end{array}$ & $\begin{array}{c}\text { Bulk density } \\
\left(\mathrm{g} / \mathrm{cm}^{3} \text {, average }\right)\end{array}$ & $\begin{array}{c}\text { Porosity (open pore) } \\
(\%)\end{array}$ & $\begin{array}{c}\text { Porosity (open pore) } \\
(\%, \text { average })\end{array}$ \\
\hline \multirow{3}{*}{ Before impregnation } & 1 & 1.30 & \multirow{3}{*}{1.29} & 30.4 & \multirow{3}{*}{29.8} \\
\hline & 2 & 1.29 & & 29.8 & \\
\hline & 3 & 1.29 & & 29.3 & \\
\hline \multirow{3}{*}{ After impregnation } & 4 & 1.44 & \multirow{3}{*}{1.44} & 25.0 & \multirow{3}{*}{25.2} \\
\hline & 5 & 1.43 & & 25.5 & \\
\hline & 6 & 1.44 & & 25.2 & \\
\hline
\end{tabular}

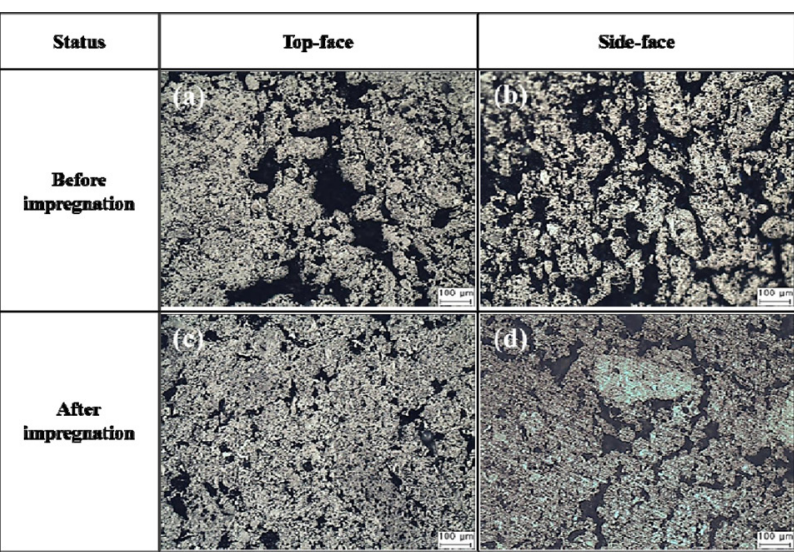

Fig. 2. Optical microscopy images $(\times 100)$ of bulk graphite before and after impregnation.

of the (002)-peak is very great, and the (100)-peak and the (101)-peak observed at the $2 \theta$ positions of 42.22 and 44.39 (JCPDS-ICDD \#411487) are clearly distinguished. This shows the outstanding crystallinity of ISGS.

\subsection{Density and porosity}

Table 1 shows the density and the porosity of the bulk graphite before and after impregnation, of the three samples measured. The density of the pre-impregnation bulk graphite and the post-impregnation density were $1.29 \mathrm{~g} / \mathrm{cm}^{3}$ and $1.44 \mathrm{~g} / \mathrm{cm}^{3}$, respectively, thus exhibiting an increase of $11.1 \%$ after impregnation. The pre-impregnation porosity was $29.8 \%$, and the postimpregnation porosity was $25.2 \%$, respectively, thus showing a decrease of $4.6 \%$. This decrease in the porosity can be seen as the result of infiltration and impregnation of resin into the open pores. Consequently, if the iteration of impregnation is increased, it is expected that high-density bulk-graphite using recycled ISGS can be produced.

\subsection{Microstructures}

Fig. 2 shows the microstructure of the bulk graphite before and after impregnation. In the microstructure of the top-face and the side-face, it was possible to observe the directionality of the

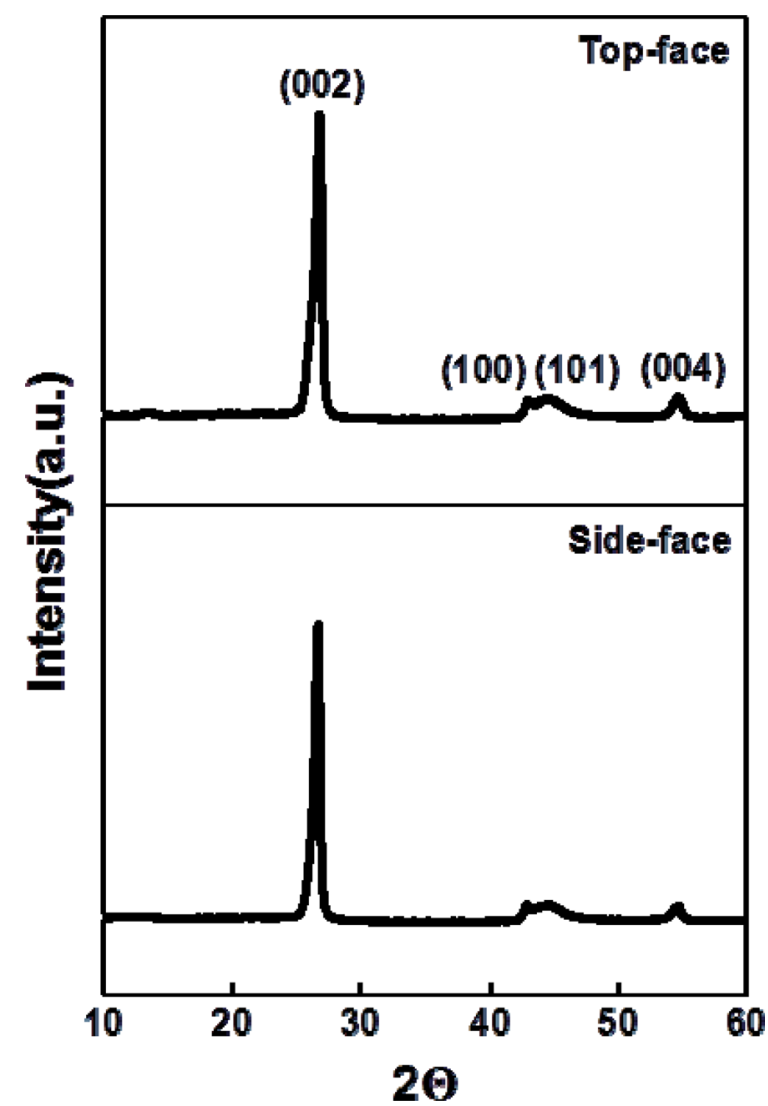

Fig. 3. X-ray diffractometer spectra of manufactured bulk graphite.

particles. Consequently, the possibility of producing isotropic bulk graphite using ISGS was confirmed. It was also possible to confirm a decrease in the pore distribution of the microstructures after impregnation and, as indicated by the data on density and porosity, to confirm an impregnation effect.

\subsection{Anisotropy ratio}

Fig. 3 shows the top-face and side-face XRD spectra of bulk graphite. It clearly shows the (002)-peak, and a distinction between the (100)-peak and the (101)-peak was confirmed. Differences in the XRD spectra between the top-face and the side-face 
Table 2. Degree of alignment and anisotropy ratio from X-ray diffractometer

\begin{tabular}{ccc} 
Status & $\begin{array}{c}\text { Degree of alignment } \\
\left(\mathrm{Da}=\mathrm{I}_{100} /\left(\mathrm{I}_{100}+\mathrm{I}_{002}\right)\right)\end{array}$ & $\begin{array}{c}\text { Anisotropy ratio } \\
\left(\mathrm{Da}^{\mathrm{Top}} / \mathrm{Da}^{\text {Side }}\right)\end{array}$ \\
\hline Top-face & 0.071 & 1.13 \\
Side-face & 0.063 & \\
\hline
\end{tabular}

were not observed. Table 2 shows the $\mathrm{Da}$ and the anisotropy ratio from the strength of the (002)-peak and the (100)-peak of the XRD spectra. The Da values of the top-face and the side-face were 0.071 and 0.063 , respectively, and the difference between them was 0.008 , thus confirming that there was no alignment in a particular direction. This also agreed with the earlier results of study of the microstructure. In addition, the anisotropy ratio calculated with the $\mathrm{Da}$ of the top-face and the side-face was 1.13, thus confirming the production of comparatively good, new, isotropic bulk-graphite [20].

\section{Conclusions}

The present study sought to produce isotropic bulk graphite using recycled ISGS, and the characteristics of the bulk graphite thus produced were studied, leading to the following conclusions:

The density and porosity of the new bulk graphite produced were $1.29 \mathrm{~g} / \mathrm{cm}^{3}$ and $29.8 \%$, respectively. After a single resin impregnation, the density rose to $1.44 \mathrm{~g} / \mathrm{cm}^{3}$ (11.1\% increase) and the porosity fell to $25.2 \%$ ( $4.6 \%$ decrease).

According to the results from examination of the microstructure, the pores in the new bulk graphite were confirmed to decrease after impregnation, but directionality of particles exposed on the top-face and side-face was not observed.

According to the results from calculations obtained using XRD spectra, the Da values were 0.071 for the top-face and 0.063 for the side-face: a difference of 0.008 , and the anisotropy ratio was 1.13: confirming that the newly produced bulk graphite was isotropic.

Consequently, the present study confirmed that it is possible to produce isotropic bulk graphite using recycled ISGS. It is expected that it will be possible in the future to produce highdensity, isotropic bulk-graphite by increasing the number of impregnation cycles.

\section{Acknowledgments}

This work was supported by the Kumoh National Institute of Technology.

\section{References}

[1] Park SM, Yasuda E, Park YD. The influence of graphitic structure on oxidation reaction of carbon materials. J Korean Ceram Soc, $\mathbf{3 3}$, 816 (1996).
[2] Liu Z, Guo Q, Shi J, Zhai G, Liu L. Graphite blocks with high thermal conductivity derived from natural graphite flake. Carbon, 46, 414 (2008). http://dx.doi.org/10.1016/j.carbon.2007.11.050.

[3] Youm HN, Kim KJ, Lee JM, Chung YJ. Effects of impregnation on the manufacture of high density carbon materials. J Korean Ceram Soc, 30, 852 (1993).

[4] Choi WK, Kim BJ, Chi SH, Park SJ. Nuclear graphites (I): oxidation behaviors. Carbon Lett, 10, 239 (2009).

[5] Jung JW, Kim KT. Effect of rubber mold on densification behavior of metal powder during cold isostatic pressing. Trans Korean Soc Mech Eng A, 22, 330 (1998).

[6] Chung SH, Kim KW, Kim MS, Lim YS. Analysis on raw materials for graphite electrode. J Res Inst Ind Technol, 18, 365 (1999).

[7] Wissler M. Graphite and carbon powders for electrochemical applications. J Power Sources, 156, 142 (2006). http://dx.doi. org/10.1016/j.jpowsour.2006.02.064.

[8] Park YD. Production technology of artificial graphite electrodes for arc furnace. Polym Sci Technol, 8, 155 (1997).

[9] Janerka K, Bartocha D. The sort of carburization and the quality of obtained cast iron. Arch Foundry Eng, 8, 55 (2008).

[10] Roessler A, Crettenand D. Direct electrochemical reduction of vat dyes in a fixed bed of graphite granules. Dyes Pigments, 63, 29 (2004). http://dx.doi.org/10.1016/j.dyepig.2004.01.005.

[11] Fan $\mathrm{Cl}$, Chen H. Preparation, structure, and electrochemical performance of anodes from artificial graphite scrap for lithium ion batteries. J Mater Sci, 46, 2140 (2011). http://dx.doi.org/10.1007/ s10853-010-5050-y.

[12] Aas KL. Performance of two graphite electrode qualities in EDM of seal slots in a jet engine turbine vane. J Mater Process Technol, 149, 152 (2004). http://dx.doi.org/10.1016/j.jmatprotec.2004.02.005

[13] Han YS, Kim HJ, Shin YS, Park JK, Ko JC. Silver coating on the porous pellets from porphyry rock and application to an antibacterial media. J Korean Ceram Soc, 46, 16 (2009).

[14] Ishizaki KZ, Komarneni S, Nanko M. Porous Materials: Process Technology and Applications, Kluwer Academic Publishers, Dordrecht (1998)

[15] Cao A, Xu C, Liang J, Wu D, Wei B. X-ray diffraction characterization on the alignment degree of carbon nanotubes. Chem Phys Lett, 344, 13 (2001). http://dx.doi.org/10.1016/S0009-2614(01)006716.

[16] Roh JS. A structural study of the oxidized high modulus pitch based carbon fibers by oxidation in carbon dioxide. Carbon Lett, $\mathbf{5}$, 27 (2004).

[17] Seehra MS, Pavlovic AS, Babu VS, Zondlo JW, Stansberry PG, Stiller AH. Measurements and control of anisotropy in ten coal-based graphites. Carbon, 32, 431 (1994). http://dx.doi. org/10.1016/0008-6223(94)90163-5.

[18] Weller TE, Ellerby M, Saxena SS, Smith RP, Skipper NT. Superconductivity in the intercalated graphite compounds $\mathrm{C} 6 \mathrm{Yb}$ and C6Ca. Nat Phys, 1, 39 (2005). http://dx.doi.org/10.1038/ nphys 0010 .

[19] Slack GA. Anisotropic thermal conductivity of pyrolytic graphite. Phys Rev, 127, 694 (1962). http://dx.doi.org/10.1103/PhysRev.127.694.

[20] Oh JK, Lee SW, Park KW. Preparation of isotropic carbon with high density. J Korean Ceram Soc, 28, 908 (1991). 